\title{
Preface to the special issue on electromagnetic phenomena related to seismic and volcanic activities from EMSEV in 2016
}

\author{
Xuemin Zhang $\cdot$ Katsumi Hattori $\cdot$ Valerio Tramutoli
}

Published online: 29 November 2017

(C) The Author(s) 2017. This article is an open access publication

EMSEV, Electromagnetic Studies of Earthquake and Volcanoes, set up in 2002, is a joint academic organization by multi-associations in IUGG (International Union of Geodesy and Geophysics), including IAGA (International Association of Geomagnetism and Aeronomy), IASPEI (International Association of Seismology and Physics of the Earth's Interior), IAVCEI (International Association of Volcanology and Chemistry of the Earth's Interior). On August 25-29, 2016, the 2016 International EMSEV Workshop on the observation and understanding of the various kinds of electromagnetic phenomena associated with earthquakes and volcanic eruptions was held in Lanzhou, Gansu Province. It is the first EMSEV meeting held in Chinese mainland. The topics of the meeting were as follows: natural and induced seismicity and volcanic activity; electromagnetic signals associated with great earthquakes and volcanoes eruptions; earthquake and volcanic eruption precursors from lithosphere to space, studies of earthquakes and volcanoes by electromagnetic methods with multi-disciplinary approaches; mechanism, laboratory, and modeling studies for active faults and volcanoes; seismo-electric, seismo-magnetic and seismo-electromagnetic observations and application in China. More than 200

X. Zhang $(\bowtie)$

Institute of Earthquake Forecasting, China Earthquake

Administration, Beijing 100036, China

e-mail: zhangxm96@126.com

\section{K. Hattori}

Graduate School of Science, Chiba University, 1-33,

Yayoi, Inage, Chiba 263-8522, Japan

V. Tramutoli

Engineering School (SI), University of Bailicata- Via Dell'

Ateneo Lucano, 10-85100 Potenza, Italy experts from 12 countries attended this conference, and 100 papers have been presented. A lot of new achievements and substantial discussions have been accomplished.

With the development of space detection technologies since last century in the world, seismo-electromagnetic monitoring has been gradually constructed to be a stereo observing system. The new results in EMSEV 2016 illustrated that, not only the traditional ground-based observations such as electric field, magnetic field, apparent resistivity, electromagnetic emissions, have been developed quickly, but also the seismo-ionospheric detection attracted more and more attention including ionosonde, VLF radio wave, Schumann resonance, and satellite electromagnetic detection. This stereo seismo-electromagnetic monitoring system will provide strong support for understanding the formation, propagation, and coupling processes of electromagnetic signals from lithosphere to atmosphere and ionosphere during earthquake preparation and occurrence. By combining the multi-parameters together from ground to the ionosphere, the earthquake preparation process can be built up not only in temporal frame, but in spatial distributions due to their different sensitivity to the physical properties of media in the Earth's spheres (Zhang and Shen 2011; Zhang et al. 2016). The development in new technologies and data processing methods on seismo-electromagnetics exhibited in EMSEV 2016 is quite inspiring.

In view of the amount of achievements in seismo-electromagnetics shown in EMSEV 2016 meeting, EMSEV commission decided to publish some of the new results in three journals in Chinese and English during the meeting, in which Earthquake Science has received 21 papers, while 5 papers have been accepted after peer-review and collected in this special issue as the first step, and about more 
than 5 paper still under revision will be published in the following issues once they are accepted.

In this special issue, some new techniques in electromagnetic field data analysis have been introduced and applied into the earthquake research. Nivokov et al. illustrated the possibility of electrical earthquake triggering based on their laboratory experiments. By using the springblock facility, their rock experiment confirmed that pulses of direct current (DC) injected along the fault area can directly trigger a seismic event, but only in the subcritical shear stress range, being $0.98-0.99$ of critical value corresponded to occurrence of sharp slip. The laboratory current density triggered the seismic event which was larger approximately by 8 orders compared with calculated one in the field experiments by magneto-hydrodynamic (MHD) generator. The mechanism of injected electric current for local seismicity was discussed, one from the current contraction in the narrow highly conductive fault zones, one being driven by electric pulses due to fluid migration into the fault zone.

Tan et al. studied the signals in spontaneous electric field related to earthquakes and verified the anomalies by the method of correlating regional synchronization contrasts (CRSC). Their statistical results presented that at least $30 \%$ of the stations exhibited trend anomalies during the strong earthquake cluster periods in China and its neighboring areas, which enhances the reliability of the spontaneous field anomalies with regional tectonic activities. It is also illustrated that CRSC method is more adaptable to the regions with complex electromagnetic environments and fault distributions, while the VAN method is more suitable for the blocks with relatively stable tectonic activities.

The paper from Varotsos et al. presented the results by using natural time analysis to identify the missed precursors before the $M_{\mathrm{W}} 9.0$ Tohoku earthquake in 2011. They found the fluctuations in seismicity, an evident minimum, occurred two months before Tohoku earthquake, which is strikingly simultaneous with the anomalous geomagnetic field variations in $z$ component, showing the similar characteristic change in seismicity and Seismic Electric Signals (SES) activity from VAN method.

Kappler et al. introduced the application of the method of time-domain principal component analysis (TDPCA) filtering into magnetometer data processing. Several types of pulses within the electromagnetic time series data have been identified and used as training datum, and the automatic process to select the isolate pulses with similar characteristics has been developed. It is found that the method of TDPCA is useful to find and separate the pulses emitted from the same source such as DC train noise and natural field events, therefore, some cultural noises can be removed from the anomaly distinguishing of earthquake research.

In another paper, Varotsos et al. summarized the recent advances on determination of the occurrence time of an impending major earthquake. They presented the procedures to identify the occurrence time of a major earthquake by using the natural time analysis method under two conditions, with or without geoelectrical data. With the SES in Greece, a preliminary procedure considering the seismicity in one subarea and the "updated" taking account of all the possible subareas have been employed, in which the updated one can include more small events and identify the subarea at critical point in this region, while the preliminary one needs to adjust the magnitude threshold to avoid the effects from big earthquakes just before the major one. Without the electric field data but geomagnetic field data available in Japan for the $M_{\mathrm{W}} 9.0$ Tohoku earthquake in 2011, it is found that the $M_{\mathrm{W}} 7.3$ foreshock on March 9, 2011, played an important role to reach the critical point in seismicity analysis. Their results illustrated that using the minimum of the fluctuations of seismicity to deduce the epicentral area of future major shock should ascertain this minimum to be truly a precursory one of a major earthquake.

Finally, as the chief editors of this special issue, three of us would like to express our sincerely appreciation to all the authors for submitting their fruitful contributions and to all the reviewers for their strict and careful evaluation, and good suggestions for improvement. We hope the authors with revised comments at present pay more attention and patience on their papers, improve continuously and complete the final publication in following issues.

We also thank the editorial board, Dr. Tian Lili and Prof. Chen Yun-tai, who gave us strong support and special patience for this special issue of EMSEV 2016.

Open Access This article is distributed under the terms of the Creative Commons Attribution 4.0 International License (http://crea tivecommons.org/licenses/by/4.0/), which permits unrestricted use, distribution, and reproduction in any medium, provided you give appropriate credit to the original author(s) and the source, provide a link to the Creative Commons license, and indicate if changes were made.

\section{References}

Zhang XM, Shen XH (2011) Electromagnetic anomalies around the Wenchuan Earthquake and their relationship with earthquake preparation. International Journal of Geophysics. https://doi.org/ 10.1155/2011/904132

Zhang XM, Shen XH, Zhao SF, Liu J, Ouyang XY, Lou WY, Zeren ZM, He JH, Qian JD (2016) The seismo-ionospheric monitoring technologies and their application research development. Acta Seismol Sin 38(3):356-375 (in Chinese with English Abstract) 